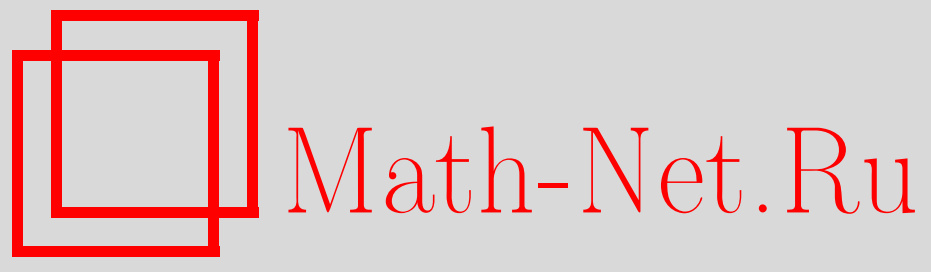

Я. Н. Шитов, О матрицах с различными тропическим рангом и рангом Капранова, Матем. заметки, 2012, том 92, выпуск 2, 316-320

DOI: https://doi.org/10.4213/mzm9273

Использование Общероссийского математического портала Math-Net.Ru подразумевает, что вы прочитали и согласны с пользовательским соглашением http://www . mathnet.ru/rus/agreement

Параметры загрузки:

IP : 34.227 .88 .159

26 апреля 2023 г., 16:37:12

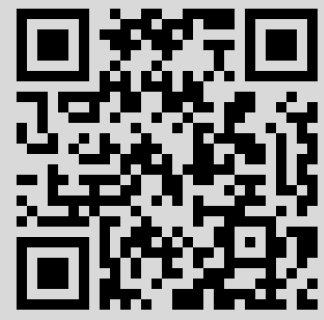




\section{О матрицах с различными тропическим рангом и рангом Капранова}

\section{Я.Н. Шитов}

Основным объектом тропической математики является тропическое полукольио, т.е. множество $\mathbb{R}$ вещественных чисел с операциями тропического сложения, $\oplus$, и тропического умножения, $\otimes$, определяемыми как $a \oplus b=\min \{a, b\}, a \otimes b=a+b$ для всех $a, b \in \mathbb{R}$. Заметим, что элемент $0 \in \mathbb{R}$ нейтрален относительно тропического умножения. Тропическая математика получается из традиционной как результат деквантования Маслова (см. [1]-[3]). Методы тропической математики оказываются полезными для многочисленных приложений (см. [4]-[6]), применяются в алгебраической геометрии (см. [7], [8]). Большой интерес вызывает понятие ранга тропических матриц (см. [4], [9], [10]). В отличие от случая матриц над полем имеется множество различных ранговых функций для тропических матриц, многие из них описаны в работах [4], [9], [10]. Данная заметка посвящена понятиям тропического ранга и ранга Капранова.

Мы будем использовать символ $\mathbb{F}$ как обозначение поля, $\mathbb{F}^{*}$ - обозначение множества ненулевых элементов $\mathbb{F}$. Через $a_{i j}$ мы обозначаем элементы матрицы $A$, через $A^{(j)}-$ ее $j$-й столбец, через $A_{(i)}-i$-ю строку, через $A^{\top}$ - матрицу, транспонированную к $A$. Подматрица матрицы $A$, образованная ее строками с номерами $r_{1}, \ldots, r_{k}$, обозначается через $A\left[r_{1}, \ldots, r_{k}\right]$, подматрица матрицы $A\left[r_{1}, \ldots, r_{k}\right]$, образованная ее столбцами с номерами $c_{1}, \ldots, c_{l}$, - через $A\left[r_{1}, \ldots, r_{k} \mid c_{1}, \ldots, c_{l}\right]$.

Через $\mathbf{H}_{\mathbb{F}}$ мы обозначаем поле (см. [11]), состоящее из формальных сумм вида $a(t)=$ $\sum_{e \in \mathbb{R}} a_{e} t^{e}$, где $t$ - переменная, коэффициенты $\left\{a_{e}\right\}$ принадлежат полю $\mathbb{F}$, а носитель $E(a)=\left\{e \in \mathbb{R}: a_{e} \neq 0\right\}$ является вполне упорядоченным подмножеством множества $\mathbb{R}$, т.е. любое непустое подмножество множества $E(a)$ содержит наименьший элемент. Степенъю элемента $a \in \mathbf{H}_{\mathbb{F}}^{*}$ называется показатель степени его главного члена, т.е. $\operatorname{deg} a=\min E(a)$. Элемент $a_{0} \in \mathbb{F}$ называется свободным иленом $а$. Степень нулевого элемента поля $\mathbf{H}_{\mathbb{F}}$ полагается равной $+\infty$. Матрица, полученная из матрицы $A \in\left(\mathbf{H}_{\mathbb{F}}^{*}\right)^{m \times n}$ поэлементным применением отображения $\operatorname{deg}$, также обозначается через $\operatorname{deg} A \in \mathbb{R}^{m \times n}$. Теперь мы можем определить понятие ранга Капранова (см. [9; следствие 3.4]).

ОПРедЕЛЕНИЕ 1. Ранг Капранова матрицы $B \in \mathbb{R}^{m \times n}$ относительно базового поля $\mathbb{F}$, $\mathrm{K}_{\mathbb{F}}(B)$, есть $\min \left\{\operatorname{rank}(A) \mid A \in\left(\mathbf{H}_{\mathbb{F}}^{*}\right)^{m \times n}, \operatorname{deg} A=B\right\}$, где $\operatorname{rank}(A)$ - классический ранг матрицы $A$ с элементами из поля $\mathbf{H}_{\mathbb{F}}$.

Тропический перманент матрицы $B \in \mathbb{R}^{n \times n}$ определяется как

$$
\operatorname{perm}(B)=\min _{\sigma \in S_{n}}\left\{b_{1, \sigma(1)}+\cdots+b_{n, \sigma(n)}\right\},
$$

где через $S_{n}$ обозначена симметрическая группа множества $\{1, \ldots, n\}$. Матрица $B$ называется тропически вырожденной, если минимум в выражении (1) достигается, по крайней мере, дважды. В противном случае матрица $B$ называется тропически невырожденной.

ОПределение 2. Тропический ранг матрицы $M \in \mathbb{R}^{d \times n}, \operatorname{trop}(M)$, есть размер ее наибольшей тропически невырожденной квадратной подматрицы.

Следующее утверждение следует непосредственно из определений.

УтвеРЖДЕние 3. Значения $\operatorname{trop}(A) u \mathrm{~K}_{\mathbb{F}}(A)$ инвариантны относителъно прибавления фиксированного числа $к$ каждому элементу некоторой строки или столбиа тропической матрицы $A$, что соответствует тропическому умножению строки или столбца этой матрицы на константу. 
Множество всех $i \in\{1, \ldots, m\}$, на которых достигается $\min _{i=1}^{m}\left\{a_{i}+b_{i}\right\}$, обозначается через $\Theta(a, b)$, где $a, b \in(\mathbb{R} \cup\{+\infty\})^{m}$. Система строк матрицы $A \in \mathbb{R}^{m \times n}$ называется mропически линейно зависимой, если найдется вектор $\lambda \in \mathbb{R}^{m}$, для которого $\left|\Theta\left(\lambda, A^{(j)}\right)\right| \geqslant 2$ при каждом $j \in\{1, \ldots, n\}$. В этом случае мы говорим, что вектор $\lambda$ реализует тропическую зависимость строк матрицы $A$. Важную роль играет следующая теорема (см. [12; теорема 5.11]).

Теорема 4. Тропический ранг матрицы равен размеру самой болъшой тропически линейно независимой подсистемы системы ее строк.

Девелин, Сантос и Штурмфельс сформулировали следующий вопрос.

Вопрос 5 [9; раздел 8 , вопрос (6)]. Существует ли матрица размера $5 \times 5$ с тропическим рангом 3 и рангом Капранова 4 ?

Чан, Йенсен и Рубеи показали (см. [13; следствие 1.5$])$, что $\operatorname{trop}(A)=\mathrm{K}_{\mathbb{C}}(A)$ для любой матрицы $A \in \mathbb{R}^{5 \times n} ;$ их результат дает ответ на вопрос 5 в случае, когда ранг Капранова понимается в смысле базового поля $\mathbb{C}$. Тем не менее, в работе [9] ранг Капранова рассматривается в случае произвольного базового поля $\mathbb{F}$, см. [9; определение 3.9]. В нашей заметке мы рассматриваем этот случай и даем общий ответ на вопрос 5. Мы показываем, что если $|\mathbb{F}| \geqslant 4$, а матрица $B \in \mathbb{R}^{5 \times n}$ такова, что $\operatorname{trop}(B) \leqslant 3$, то $\mathrm{K}_{\mathbb{F}}(B) \leqslant 3$. Кроме того, мы приводим примеры матриц размера $5 \times 5$ с тропическим рангом 3 и рангом Капранова 4 в случае базовых полей $\mathbb{F}_{2}$ и $\mathbb{F}_{3}$. Докажем следующие леммы, полезные для доказательства основного результата.

Лемма 6. Пусть $|\mathbb{F}| \geqslant 4, S \in \mathrm{H}_{\mathbb{F}}^{2 \times 2}$. Тогда найдется $\xi \in \mathbb{F}^{*}$, для которого

$$
\operatorname{deg}\left(\xi s_{i 1}+s_{i 2}\right)=\min \left\{\operatorname{deg} s_{i 1}, \operatorname{deg} s_{i 2}\right\} \quad \text { npu } i \in\{1,2\} .
$$

ДокАЗАТЕльСтво. Если $s_{i j} \neq 0$, то через $\sigma_{i j}$ мы обозначим коэффициент главного члена элемента $s_{i j}$. Если же $s_{i j}=0$, определим $\sigma_{i j} \in \mathbb{F}^{*}$ произвольно. Теперь достаточно выбрать $\xi \in \mathbb{F} \backslash\left\{0,-\sigma_{12} / \sigma_{11},-\sigma_{22} / \sigma_{21}\right\}$.

Лемма 7. Пусть $|\mathbb{F}| \geqslant 4$. Пусть матрица $A \in \mathbf{H}_{\mathbb{F}}^{5 \times 2}$ такова, что $\operatorname{rank}(A)=2 u$

$$
\operatorname{deg}\left(a_{p 1} a_{q 2}-a_{q 1} a_{p 2}\right)=\min \left\{\operatorname{deg} a_{p 1}+\operatorname{deg} a_{q 2}, \operatorname{deg} a_{q 1}+\operatorname{deg} a_{p 2}\right\}
$$

для любых различных $p, q \in\{1, \ldots, 5\}$. Пусть $B \in \mathbb{R}^{5 \times n}$, положим

$$
\Theta_{1 j}=\Theta\left(\operatorname{deg} A^{(1)}, B^{(j)}\right), \quad \Theta_{2 j}=\Theta\left(\operatorname{deg} A^{(2)}, B^{(j)}\right) \quad \text { при всех } \quad j \in\{1, \ldots, n\} .
$$

Пусть $\left|\Theta_{1 j}\right| \geqslant 2,\left|\Theta_{2 j}\right| \geqslant 2,\left|\Theta_{1 j} \cup \Theta_{2 j}\right| \geqslant 3$ при любом $j$. Тогда $\mathrm{K}_{\mathbb{F}}(B) \leqslant 3$.

ДоказАТЕЛЬСтво. Фиксируем произвольное $j \in\{1, \ldots, n\}$, обозначим

$$
\theta_{1}=\min _{i=1}^{5}\left\{\operatorname{deg} a_{i 1}+b_{i j}\right\}, \quad \theta_{2}=\min _{i=1}^{5}\left\{\operatorname{deg} a_{i 2}+b_{i j}\right\} .
$$

Условия леммы позволяют считать, не ограничивая общности, что $1 \in \Theta_{1 j}, 2 \in \Theta_{2 j}$, а также, что $\Theta_{1 j}$ и $\Theta_{2 j}$ имеют непустые пересечения с множеством $\{3,4,5\}$. В этом случае

$$
\min _{\iota=3}^{5}\left\{\operatorname{deg} \operatorname{det} A[1, \iota]+b_{1 j}+b_{\iota j}\right\}=\min _{\iota=3}^{5}\left\{\operatorname{deg} \operatorname{det} A[2, \iota]+b_{2 j}+b_{\iota j}\right\}=\theta_{1}+\theta_{2} .
$$

Из леммы 6 теперь следует, что при некоторых $\xi_{3}, \xi_{4}, \xi_{5} \in \mathbb{F}^{*}$ верно, что

$$
\operatorname{deg}\left(\sum_{\iota=3}^{5} \operatorname{det} A[1, \iota] t^{b_{1 j}+b_{\iota j}} \xi_{\iota}\right)=\operatorname{deg}\left(\sum_{\iota=3}^{5} \operatorname{det} A[2, \iota] t^{b_{2 j}+b_{\iota j}} \xi_{\iota}\right)=\theta_{1}+\theta_{2} .
$$


Из формул Крамера следует, что решение $\left(x_{1}, x_{2}\right)$ системы уравнений

$$
\left\{\begin{array}{l}
a_{11} t^{b_{1 j}} x_{1}+a_{21} t^{b_{2 j}} x_{2}=\sum_{\iota=3}^{5} \xi_{\iota} a_{\iota 1} t^{b_{\iota j}} \\
a_{12} t^{b_{1 j}} x_{1}+a_{22} t^{b_{2 j}} x_{2}=\sum_{\iota=3}^{5} \xi_{\iota} a_{\iota 2} t^{b_{\iota j}}
\end{array}\right.
$$

таково, что выполнено $\operatorname{deg} x_{1}=\operatorname{deg} x_{2}=0$. Положим $c_{1 j}=x_{1} t^{b_{1 j}}, c_{2 j}=x_{2} t^{b_{2 j}}, c_{\iota j}=-\xi_{\iota} t^{b_{\iota j}}$ при $\iota \in\{3,4,5\}$. Равенства (3) показывают, что $\sum_{i=1}^{5} a_{i 1} c_{i j}=\sum_{i=1}^{5} a_{i 2} c_{i j}=0$. Пользуясь произвольностью выбора $j \in\{1, \ldots, n\}$, строим матрицу $C$ такую, что $B=\operatorname{deg} C$, а строки $\sum_{i=1}^{5} a_{i 1} C_{(i)}$ и $\sum_{i=1}^{5} a_{i 2} C_{(i)}$ нулевые. Из определения 1 теперь следует, что $\mathrm{K}_{\mathbb{F}}(B) \leqslant 3$.

Лемма 8. Если $|\mathbb{F}| \geqslant 4$, элементы матрицы $B \in \mathbb{R}^{5 \times n}$ неотрицательны, а каждый ее столбеи, содержит не менее трех нулей, то $\mathrm{K}_{\mathbb{F}}(B) \leqslant 3$.

ДоказАтЕЛЬСтво. Выберем различные $\eta, \zeta \in \mathbb{F} \backslash\{0,1\}$ и положим

$$
A=\left(\begin{array}{lllll}
1 & 1 & 1 & 1 & 0 \\
1 & \eta & \zeta & 0 & 1
\end{array}\right)^{\top} \in \mathbf{H}_{\mathbb{F}}^{5 \times 2} .
$$

Теперь результат следует из леммы 7 .

Лемма 9. Пусть элементы матрицы $B \in \mathbb{R}^{5 \times n}$ неотрицательны и

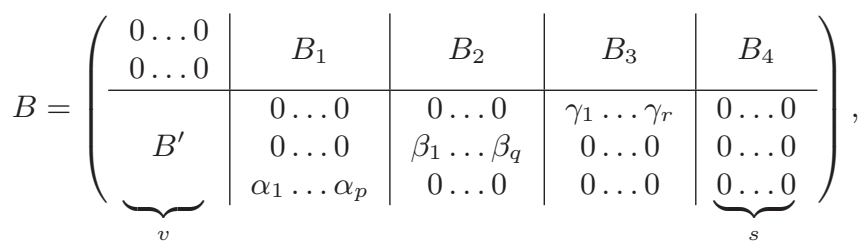

где $v>0, p>0, q+r+s>0$, какая-то из матрии, $B^{\prime}$ u $\left(B_{1}|\ldots| B_{4}\right)$ состоит из положительных элементов, числа $\alpha_{1}, \ldots, \alpha_{p}, \beta_{1}, \ldots, \beta_{q}, \gamma_{1}, \ldots, \gamma_{r}$ положительны. Пусть $|\mathbb{F}| \geqslant 4$, $\operatorname{trop}(B) \leqslant 3$. Тогда $\mathrm{K}_{\mathbb{E}}(B) \leqslant 3$.

ДокАЗАТЕЛЬСТво. От противного.

1. Предположим, что матрица $B$ удовлетворяет условию леммы и такова, что $\mathrm{K}_{\mathbb{F}}(B)>3$. Можно считать, не ограничивая общности, что значение $p+q+r+s$ минимально среди всех матриц с таким свойством.

2. Обозначим минимальный элемент матрицы $\left(B_{1}|\ldots| B_{4}\right)$ через $m$. Прибавим $-m$ к каждому элементу первых двух строк матрицы $B, m-$ к каждому элементу первых $v$ столбцов. Таким образом, согласно утверждению 3 можно считать, не ограничивая общности, что матрица $B^{\prime}$ состоит из положительных элементов и $m=0$.

3. Пусть каждая из матриц $B_{2}$ и $B_{3}$ содержит столбец без нулей (номера этих столбцов обозначим через $j_{1}$ и $j_{2}$ ). Пункты 1 и 2 показывают, что для некоторого $j_{3} \in\{1, \ldots, n\}$ верно, что или $b_{1 j_{3}}=0, b_{2 j_{3}}>0$, или $b_{1 j_{3}}>0, b_{2 j_{3}}=0$. Заметим, что в этом случае матрица $B\left[1,2,3,4 \mid 1, j_{1}, j_{2}, j_{3}\right]$ тропически невырождена. Из определения 2 следует, что $\operatorname{trop}(B) \geqslant 4$, что противоречит условию леммы. Таким образом, можно считать, не ограничивая общности, что любой столбец матрицы $B_{3}$ содержит нулевой элемент.

4. Согласно теореме 4 найдутся $\left(\lambda_{1}, \lambda_{2}, \lambda_{4}, \lambda_{5}\right),\left(\mu_{1}, \mu_{3}, \mu_{4}, \mu_{5}\right) \in \mathbb{R}^{4}$, реализующие тропическую зависимость строк матриц $B[1,2,4,5]$ и $B[1,3,4,5]$ соответственно. Положим $\Lambda=\left(\lambda_{1}, \lambda_{2},+\infty, \lambda_{4}, \lambda_{5}\right), M=\left(\mu_{1},+\infty, \mu_{3}, \mu_{4}, \mu_{5}\right)$, имеем $\Theta\left(\Lambda, B^{(j)}\right) \geqslant 2, \Theta\left(M, B^{(j)}\right) \geqslant 2$ для любого $j \in\{1, \ldots, n\}$. Из равенства (4) тогда следует, что $\lambda_{1}=\lambda_{2} \leqslant \min \left\{\lambda_{4}, \lambda_{5}\right\}$, 
$\mu_{3}=\mu_{4} \leqslant \mu_{5}, \mu_{4}<\mu_{1}$. Теперь легко видеть, что $\left|\Theta\left(\Lambda, B^{(j)}\right) \cup \Theta\left(M, B^{(j)}\right)\right| \geqslant 3$ для каждого $j \in\{1, \ldots, n\}$. Наконец, положим

$$
A=\left(\begin{array}{ccccc}
t^{\lambda_{1}} & t^{\lambda_{2}} & 0 & t^{\lambda_{4}} & t^{\lambda_{5}} \\
t^{\mu_{1}} & 0 & t^{\mu_{3}} & t^{\mu_{4}} & \eta t^{\mu_{5}}
\end{array}\right)^{\top} \in \mathbf{H}_{\mathbb{F}}^{5 \times 2},
$$

где $\eta \in \mathbb{F} \backslash\{0,1\}$. Доказательство завершается применением леммы 7.

Теперь мы можем доказать один из основных результатов нашей заметки.

Tеорема 10. Пусть $C \in \mathbb{R}^{5 \times n}, \operatorname{trop}(C) \leqslant 3,|\mathbb{F}| \geqslant 4$. Тогда $\mathrm{K}_{\mathbb{F}}(C) \leqslant 3$.

ДокАЗАТЕЛЬСтво. 1. Из теоремы 4 следует, что строки матрицы $C$ тропически линейно зависимы. Применяя утверждение 3 , считаем, не ограничивая общности, что матрица $C$ состоит из неотрицательных чисел и каждый ее столбец содержит, по крайней мере, два нуля.

2. Пусть минимальный элемент $i$-й строки матрицы $C$ равен $h_{i}$. При каждом $i \in$ $\{1, \ldots, 5\}$ прибавим $(-h)$ к каждому элементу $i$-й строки матрицы $C$, полученную матрицу обозначим через $B$. Теперь каждая строка матрицы $B$ содержит, по крайней мере, один нуль. В силу пункта 1 , элементы матрицы $B$ неотрицательны и каждый ее столбец содержит, по крайней мере, два нуля.

3. В силу утверждения $3 \mathrm{~K}_{\mathbb{F}}(B)=\mathrm{K}_{\mathbb{F}}(C), \operatorname{trop}(B) \leqslant 3$.

4. Если в каждом столбце матрицы $B$ содержится по крайней мере три нуля, то согласно лемме $8 \operatorname{trop}(B) \leqslant 3$. Считаем далее, что $b_{11}=b_{21}=0$, а элементы $b_{31}, b_{41}, b_{51}$ положительны. Возможны три случая.

Случай 1. Пусть некоторый столбец матрицы $B[3,4,5]$ содержит ровно один нулевой элемент. Считаем для определенности, что $b_{32}=0, b_{42}>0, b_{52}>0$. Предположим, что $b_{i^{\prime} j^{\prime}}=0, b_{i^{\prime \prime} j^{\prime}}>0$ для некоторых $i^{\prime}, i^{\prime \prime} \in\{4,5\}, j^{\prime} \in\{1, \ldots, n\}$. Согласно пункту 2 существует $j^{\prime \prime} \in\{1, \ldots, n\}$, для которого $b_{i^{\prime \prime} j^{\prime \prime}}=0$. Заметим, что матрица $B\left[2,3,4,5 \mid 1,2, j^{\prime}, j^{\prime \prime}\right]$ тропически невырождена, т.е. $\operatorname{trop}(B) \geqslant 4$, что противоречит пункту 3.

Противоречие показывает, что для любого $j \in\{1, \ldots, n\}$ верно или $b_{4 j}=b_{5 j}=0$, или $b_{4 j}, b_{5 j}>0$. В этом случае матрица $B$ удовлетворяет условиям леммы 9 с точностью до перестановки строк и столбцов.

Случай 2. Если некоторый столбец матрицы $B[3,4,5]$ содержит ровно два нуля, и нет столбца, содержащего ровно один нуль, то матрица $B$ удовлетворяет условиям леммы 9 с точностью до перестановки столбцов.

Случай 3. Наконец, предположим, что для любого $j \in\{1, \ldots, n\}$ верно или $b_{3 j}=b_{4 j}=$ $b_{5 j}=0$, или $b_{3 j}, b_{4 j}, b_{5 j}>0$. Рассмотрим множество $G$, состоящее из всех $j$, для которых среди элементов $b_{3 j}, b_{4 j}, b_{5 j}$ есть различные. Если $G$ пусто, то строки матрицы $B[3,4,5]$ совпадают, и тогда $\mathrm{K}_{\mathbb{F}}(B) \leqslant 3$.

Если же $G$ непусто, положим $m=\min \bigcup_{g \in G}\left\{b_{3 g}, b_{4 g}, b_{5 g}\right\}$. Прибавим $\left(-\min \left\{m, b_{3 j}\right\}\right)$ к каждому элементу $j$-го столбца матрицы $B$ (при всех $j=1, \ldots, n)$, прибавим $m$ к каждому элементу первых двух строк матрицы $B$. Заметим, что полученная матрица удовлетворяет либо условиям леммы 8 , либо случая 1 , либо случая 2 с точностью до перестановки столбцов. Согласно утверждению 3 полученная матрица имеет такие же тропический ранг и ранг Капранова, как матрица $B$.

Итак, $\mathrm{K}_{\mathbb{F}}(B) \leqslant 3$. В силу пункта $3 \mathrm{~K}_{\mathbb{F}}(C) \leqslant 3$. Теорема доказана.

Покажем теперь существенность условия $|F| \geqslant 4$ в теореме 10. 
ПримеР 11. Пусть

$$
B=\left(\begin{array}{lllll}
1 & 0 & 0 & 0 & 1 \\
0 & 1 & 0 & 0 & 1 \\
0 & 0 & 1 & 0 & 1 \\
0 & 0 & 0 & 0 & 1 \\
1 & 1 & 1 & 1 & 0
\end{array}\right), \quad D=\left(\begin{array}{ccccc}
1 & 0 & 0 & 0 & 1 \\
0 & 1 & 0 & 0 & 1 \\
0 & 0 & 0 & 0 & 1 \\
0 & 0 & 0 & 0 & 1 \\
1 & 1 & 1 & 1 & 0
\end{array}\right)
$$

Тогда $\operatorname{trop}(B)=\operatorname{trop}(D)=3, \mathrm{~K}_{\mathbb{F}_{3}}(B)=\mathrm{K}_{\mathbb{F}_{2}}(D)=4$.

ДоказАтельство. Применяя определение 2 , получаем $\operatorname{trop}(B)=\operatorname{trop}(D)=3$. Заметим, что если матрица $C \in \mathbf{H}_{\mathbb{F}_{2}}^{5 \times 5}$ такова, что $D=\operatorname{deg} C$, то $\operatorname{deg} \operatorname{det} C[1,2,3,5 \mid 1,2,3,5]=0$. Таким образом, $\mathrm{K}_{\mathbb{F}_{2}}(D) \geqslant 4$. $\mathrm{C}$ другой стороны, матрица $D$ содержит повторяющиеся строки, поэтому, на самом деле, $\mathrm{K}_{\mathbb{F}_{2}}(D)=4$.

Пусть матрица $A^{\prime} \in \mathbf{H}_{\mathbb{F}_{3}}^{5 \times 5}$ такова, что $B=\operatorname{deg} A^{\prime}$. Умножая строки и столбцы матрицы $A^{\prime}$ на элементы нулевой степени, будем считать, что если $i=4$ или $j=4$, то $a_{i j}^{\prime}=t^{b_{i j}}$. Заметим, что если $\operatorname{det} A^{\prime}[p, q, 4,5 \mid p, q, 4,5]=0$ при любых различных $p, q \in\{1,2,3\}$, то элементы $a_{p q}$ имеют свободные члены, равные -1 . В этом случае $\operatorname{deg} \operatorname{det} A^{\prime}[1,2,3,5 \mid 1,2,3,5]=0$. Таким образом, $\mathrm{K}_{\mathbb{F}_{3}}(B) \geqslant 4$. С другой стороны, положив $a_{i j}=t^{b_{i j}}$ при $(i, j) \in\{1,2,3,4,5\}^{2} \backslash$ $\{(4,2),(4,3)\}$ и $a_{42}=a_{43}=2+2 t$, заметим, что строка $A_{(2)}+A_{(3)}+A_{(4)}$ нулевая и $\operatorname{deg} A=B$. Согласно определению $1 \mathrm{~K}_{\mathbb{F}_{3}}(B)=4$.

Теперь мы можем дать полный ответ на вопрос 5 .

Tеорема 12. Матрица $B \in \mathbb{R}^{5 \times 5}$, для которой $\operatorname{trop}(B)=3 u \mathrm{~K}_{\mathbb{F}}(B)=4$, существует в том и только том случае, если $|\mathbb{F}| \leqslant 3$.

ДоказАтельство. Следует из теоремы 10 и примера 11.

Автор благодарен своему научному руководителю профессору А. Э. Гутерману за постоянное внимание к работе и полезные обсуждения.

\section{СПИСОК ЦИТИРОВАННОЙ ЛИТЕРАТУРЫ}

[1] Г. Л. Литвинов, Теория представлений, динамические системы, комбинаторные и алгоритмические методы. ХІІІ, Зап. научн. сем. ПОМИ, 326, ПОМИ, СПб., 2005, 145-182. [2] G. L. Litvinov, V. P. Maslov, Idempotency, Publ. Newton Inst., 11, Cambridge Univ. Press, Cambridge, 1998, 420-443. [3] В.П. Маслов, УМH, 42:3 (1987), 39-48. [4] M. Akian, S. Gaubert, A. Guterman, Tropical and Idempotent Mathematics, Contemp. Math., 495, Amer. Math. Soc., Providence, RI, 2010, 1-38. [5] F. L. Baccelli, G. Cohen, G. J. Olsder, J. P. Quadrat, Synchronization and Linearity. An Algebra for Discrete Event Systems, Wiley Ser. Probab. Math. Statist. Probab. Math. Statist., John Wiley \& Sons, Chichester, 1992. [6] B. Heidergott, G. J. Olsder, J. van der Woude, Max Plus at Work. Modeling and Analysis of Synchronized Systems: A Course on Max-Plus Algebra and its Applications, Princeton Ser. Appl. Math., Princeton Univ. Press, Princeton, NJ, 2006. [7] M. Einsiedler, M. Kapranov, D. Lind, J. Reine Angew. Math., 601 (2006), 139-157. [8] G. Mikhalkin, "Amoebas of algebraic varieties and tropical geometry", Different Faces of Geometry, Int. Math. Ser. (N. Y.), 3, Kluwer Acad. Publ., New York, 2004, 257-300. [9] M. Develin, F. Santos, B. Sturmfels, Combinatorial and Computational Geometry, Math. Sci. Res. Inst. Publ., 52, Cambridge Univ. Press, Cambridge, 2005, 213-242. [10] L. B. Beasley, A. E. Guterman, J. Korean Math. Soc., 42:2 (2005), 223-241. [11] B. Poonen, Enseign. Math. (2), 39:1-2 (1993), 87-106. [12] Z. Izhakian, Tropical and Idempotent Mathematics, Contemp. Math., 495, Amer. Math. Soc., Providence, RI, 2010, 173-191. [13] M. Chan, A. N. Jensen, E. Rubei, Linear Algebra Appl., 435:7 (2011), 1598-1611.

\section{Я.Н. Шитов}

